\title{
RESECTION FOR PULMONARY TUBERCULOSIS
}

\author{
By W. P. Cleland, M.R.C.P., F.R.C.S. \\ Surgeon, Brompton Chest Hospital, Thoracic Surgeon, King's College Hospital \\ and Postgraduate Medical School of London
}

Removal of tuberculous organs or tissues has long been an accepted and successful form of treatment in 'surgical' tuberculosis. Lesions in the kidneys, the genital organs in both sexes, cervical lymph glands and in certain bones and joints have all been treated in this way with good or excellent results even before the advent of streptomycin.

The lung, however, has until recently defied such treatment. Deliberate attempts were made earlier to resect tuberculous lungs in certain desperate situations, and some tuberculous lesions were resected unwittingly in mistake for other conditions. With few exceptions, these early resections ended in disaster with the development of broncho-pleural fistulae and tuberculous infection of the remaining lung, the pleura and the wound.

However, during the last decade considerable progress was made in thoracic surgery, much of which had a direct bearing on the problem of resecting the tuberculous lung.

The most important of these changes are:-

I. The development of the technique of dissection pneumonectomy and lobectomy with the isolation and individual treatment of the various hilar structures. This resulted in a more exact knowledge of the anatomy of the lung hilum which in turn paved the way for segmental resections.

2. Improved anaesthetic methods which enabled the anaesthetist to prevent the aspiration of infected tuberculous material from the diseased into the normal healthy areas of lung. Cuffed tubes, intrabronchial blockers and the use of posture (face down position) now provide adequate control of secretions in the average case.

3. A better appreciation of the part that dead space plays in determining the onset or persistence of pleural infection. Early elimination of dead space after resection is one of the best possible safeguards against the development of pleural infection. Carefully placed drainage tubes, the use of suction drainage, the employment of phrenic paralysis with or without a pneumoperitoneum and thoracoplasty all encourage the rapid and complete obliteration of the residual space.

4. Streptomycin. Probably the most important single factor which enabled resections to be undertaken safely was the introduction of streptomycin. This drug is now used extensively in preparing for operation, as an 'umbrella' during and after operation, and for the control of some of the postoperative complications.

There is a marked difference between the complication rates of cases treated before and those treated after the introduction of streptomycin, even though the former are a relatively small group and represent the earlier cases to be treated by resection. Recent work suggests that the complication rate for streptomycin resistant cases is significantly higher than for those with sensitive organisms.

The efficiency and safety of streptomycin is increased by the simultaneous exhibition of P.A.S. or isoniazid and one or other of these drugs should always be administered with streptomycin if only to diminish the chance of streptomycin resistance developing.

During the past five or six years resections have been used in the treatment of tuberculous lesions on an ever wider scale. (See Table 3). It has been amply demonstrated that the operation is feasible in many cases and can be carried out with a low mortality and little morbidity. The surgical and technical side of the problem seems to be on a sound basis. There still remains uncertainty, however, concerning (I) the indications for resection. (2) the late progress of patients submitted to resection and how these compare with other well established forms of treatment particularly thoracoplasty. (3) the effects on immunity of removing the whole or the greater part of the tuberculous disease from a patient. (4) the importance of antibiotics used in conjunction with surgery. 
TABLE I

Case Classification in Relation to Operation and Hospital Mortality

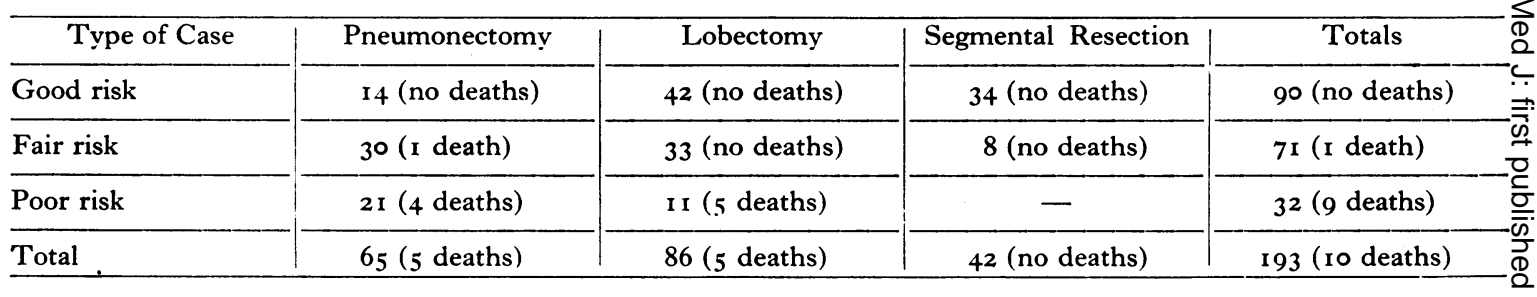

TABI.E 2

Operations with Mortality and Complication Rates

\begin{tabular}{|c|c|c|c|c|c|c|}
\hline & Totals & $\begin{array}{l}\text { Hospital } \\
\text { mortality }\end{array}$ & $\begin{array}{c}\text { Late } \\
\text { mortality }\end{array}$ & Fistula & $\begin{array}{c}\text { Empyema } \\
\text { without fistula }\end{array}$ & $\begin{array}{c}\text { Late } \\
\text { reactivation }\end{array}$ \\
\hline Pneumonectomy .. & 65 & 5 & 10 & $\mathbf{I}$ & 3 & 4 \\
\hline Lobectomy & 86 & 5 & $\mathbf{I}$ & 4 & $\mathbf{I}$ & 9 \\
\hline Segmental resection & 42 & $\circ$ & $\mathbf{I}$ & 2 & 2 & 4 \\
\hline
\end{tabular}

TABLE 3

Biennial Record of Operations with Hospital (H.) and Later (L.) Mortalities

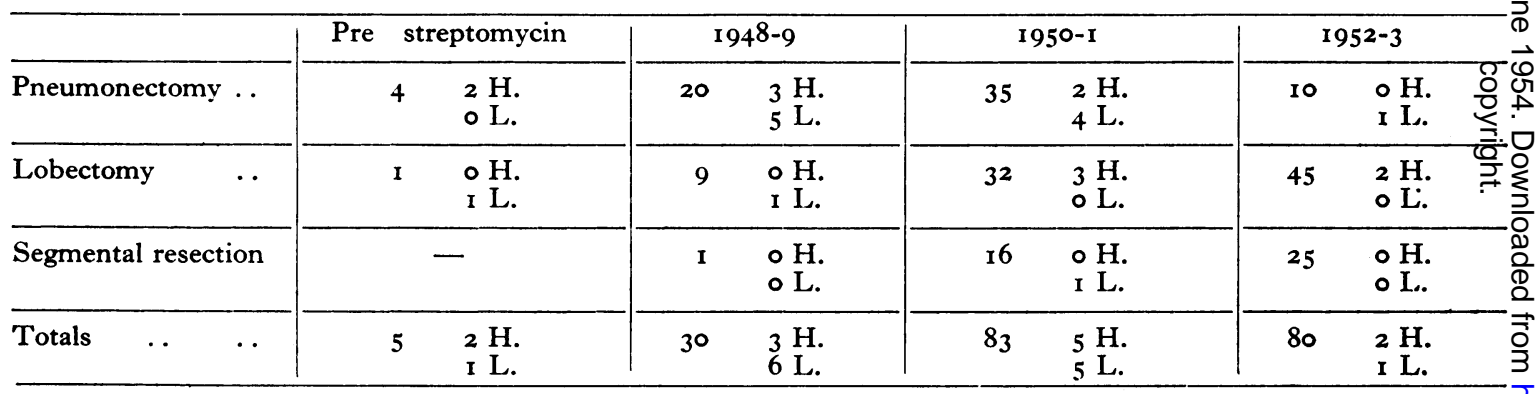

\section{The Advantages and Disadvantages of Resection}

The possible advantages of resection are (I) the extirpation of the more obvious and active tuberculous lesions with the removal of 'toxic foci ' should leave the body the less formidable task of controlling the remaining disease. This falls into line with the impression that the removal of major foci of disease frequently has a beneficial effect upon minor foci. (2) The function of remaining lung tissue is not impaired to the same extent as occurs in collapse therapy, particularly after thoracoplasty, as there is less interference with the chest wall and its movements. (3) There is no fear of unclosed cavities or residual bronchiectasis which sometimes occur after thoracoplasty. (Cavities persist in some 10 to 15 per cent. of thoracoplasties and plombs.) (4) In certain cases which are undiagnosed or unproven, resection is advisable in case the lesion is due to a more serious condition such as a carcinoma. This particularly applies to thr solitary rounded lesions.

Against this we have to consider the following potential disadvantages.

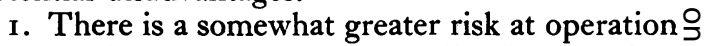
and a higher post-operative complication rate than $D$ occurs with thoracoplasty.

2. Post-operative complications, when they don occur, are often of a more serious nature (e.g., broncho-pleural fistula and empyema) and are 0 more likely to end in disaster.

3. Uncertainty about the stability of the patient from a long-term point of view. Enough timeo has not yet elapsed to provide the answer to this question. (Thoracoplasty cases show remarkable ${ }^{+}$

stability over the years.)
4. Problems created by the production of a dead $\frac{0}{\vec{D}}$ space following removal of lung tissue. 
(a) Persistence of a dead space (pleural) may result in the development of an empyema.

(b) If remaining lung tissue over-expands in an attempt to close the space, re-activation of latent tuberculous foci in the lung may occur.

5. A surgeon may be driven, for technical or pathological reasons, to remove more lung tissue than was originally intended or desired with the possible sacrifice of normal lung tissue, and in border line cases the risk of producing a respiratory cripple.

6. Inadvisability of carrying out a resection if there is active disease in any of the lung tissue which is to be left behind. This really limits resection to patients who have unilateral uncontrolled disease.

\section{Indications for Resection}

In the management of a tuberculous patient it is usual to see what can be achieved by bed rest and chemotherapy before embarking on more elaborate measures. This enables the clinician to assess the patient's powers of resistence and his ability to fight his own disease; this information may often be culled from a careful study of the clinical course and serial radiographs in chronic cases, but in others it can only be obtained after a period of observation and therapeutic trial.

With this information available the clinician is better able to decide whether further measures are necessary to control the disease bearing in mind such factors as temperament, occupation and social conditions.

In the past minor collapse measures, such as artificial pneumothorax, phrenic paralysis and pneumoperitoneum were initially employed with some measure of success and only when these failed to control the lesion or were contra-indicated were the major surgical methods of collapse therapy employed.

The standard procedure in such instances was the thoracoplasty. This operation has been in use for many years and has stood the test of time. Not only does it give a high percentage of good results with regard to cavity closure and sputum conversion, but the long term stability of the patient after operation is well known. Extrapleural pneumothorax and plombage are essentially variations of a thoracoplasty designed to overcome some of the disadvantages of the latter or to extend its indications.

It is well known that there are certain types of disease which do not do well with thoracoplasty, and some of these can now be treated by resection with more satisfactory results. The majority of clinicians are in agreement about these cases and they can be classed as the absolute indications for resection.

There remain, however, a large number of cases which could equally well be treated by either collapse therapy or resection, constituting a group of relative indications for resection. In this group the decision to resect depends largely upon the bias of the particular surgeon or physician concerned. The immediate results are fairly comparable which ever method is employed. Much will depend on whether the long-term results ( 5 to Io years) are better in one or other group.

There remains a group where resections are contra-indicated but where collapse therapy is still practicable and finally an all too large group where no radical treatment is possible.

I will now discuss the indications for resection from the point of view of the local lesion alone. However, it must be emphasised that this is not the only consideration in planning treatment and that lesions elsewhere in the lungs, other pathological conditions such as emphysema, asthma, cardiovascular disease etc., and particularly a poor respiratory reserve all come into the picture and may force one to carry out treatment which is not ideal but is either safer or more practicable.

\section{Absolute Indication for Resection}

I. Solid-Tuberculous Lesions. 'Tuberculoma' (Figures $I$ and 2.)

This is a group comprising several differing pathological entities (progressive primary lesion; circumscribed areas of caseous pneumonia-the true tuberculoma; blocked tuberculous cavities and tuberculous abscesses of the bronchus distal to a bronchial occlusion). They are, however, a radiological entity and are for convenience, therefore, grouped together as the diagnosis is usually only made after removal of the lesion. In certain instances a firm diagnosis of tuberculosis cannot be made and in such cases cancer is a possibility which should demand resection.

It is generally accepted that a lesion of this type which is larger than two centimetres in diameter should be removed. This is based on the assumption that the body cannot absorb and heal a lesion of this size and at best can only encapsulate the lesion. There are moderate risks of breakdown, excavation and spread.

\section{Lesions Associated with Broncho-Stenosis}

Many tuberculous lesions are either essentially due to, or complicated by relative or absolute stenosis of the related bronchi. The stenosis may prevent adequate healing and it is probable that collapse therapy will only serve to aggravate the stenosis. If healing of the tuberculous process has occurred, residual bronchiectasis due to the 


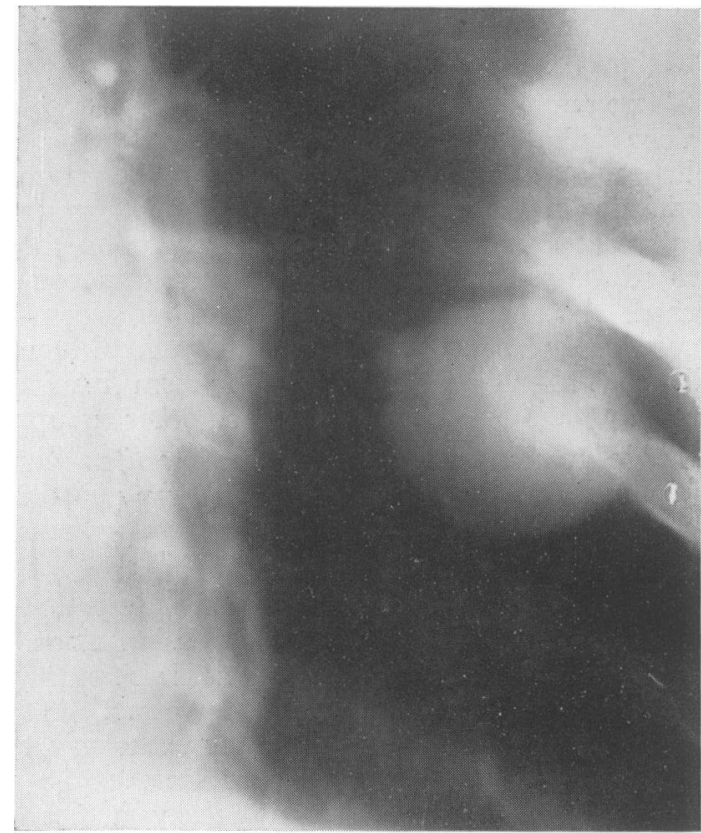

FIG. I (a)

FIG. I.-Lobectomy for a solid lesion-so-called 'Tuberculoma.' (a) P.A. tomogram through the ' tumour.' Neither calcification, cavitation, nor satellite nodules present to indicate the correct aetiology. (b) Specimen shows a solid tuberculous lesion exhibiting concentric layers or 'onion' effect and calcification in adjacent hilar glands indicating that the lesion is a progressive primary focus.

obstruction is likely, and may well produce symptoms and require treatment.

The presence of active tuberculous disease of the bronchus at the site of division may mitigate against satisfactory healing of the stump and determine the occurrence of a bronchial fistula. In cases where such disease is suspected or demonstrated, it is advisable to administer a long preoperative course of streptomycin etc., in order to encourage healing of the bronchial lesion before embarking on a resection.

Stenosis may occur in any part of the bronchial tree from the periphery to the main bronchus, or even the trachea. Stenosis of small bronchi will give rise to caseous abscess formation in that part of the bronchus beyond the obstruction. (Bronchial cold abscess.) This will appear radiographically as a round or oval solid focus but it is often possible to see the thickened bronchus leading to the solid focus.

More proximal lesions will produce segmental, lobar or total atelectasis which is readily recognized radiologically. When the larger bronchi are

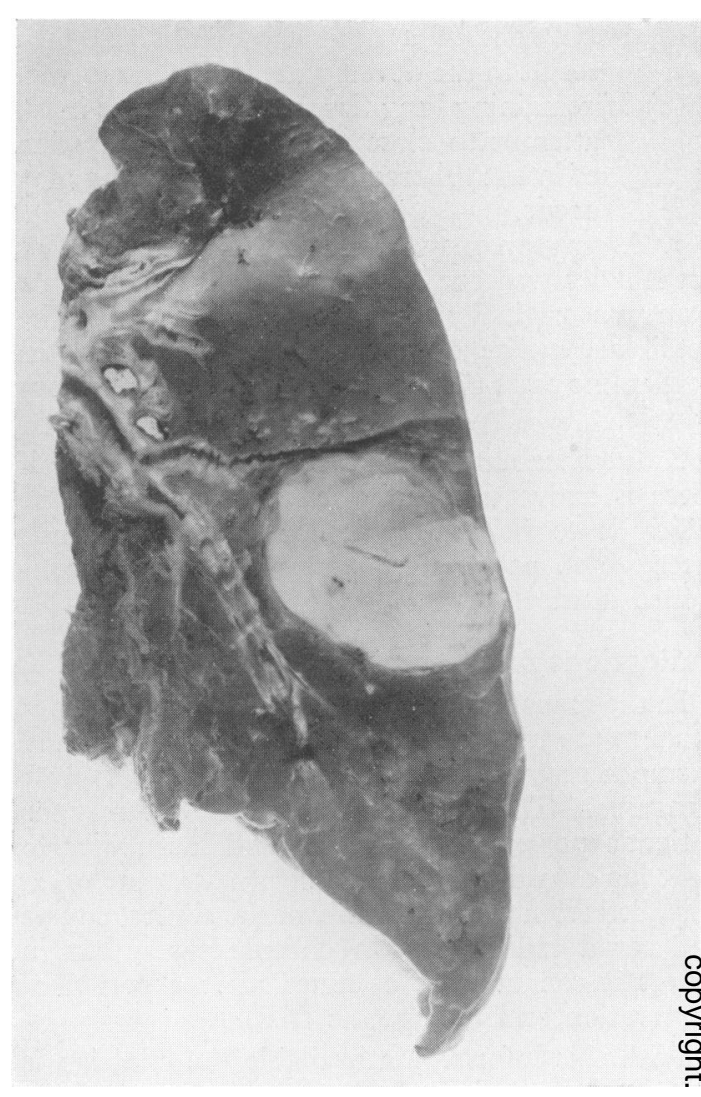

Fig. I (b)

involved, greater amounts of lung tissues are $\overline{\overline{\bar{O}}}$ rendered airless with defective bronchial drainage $\vec{\supset}$ and so in this group we find a significent increase in secondary septic complications in the involved lung. These may be severe enough to dominate the whole clinical picture and mask the essentially tuberculous nature of the condition.

Wheezing, paroxysmal coughing, persistent $\delta$ positivity of the sputum without adequate explanation should all suggest the presence of a bronchial 을 lesion but cases are found who present none of $D$ these features. Bronchoscopy is an essential o investigation and may reveal marked redness and $\mathrm{N}$ swelling of the bronchial mucosa, ulceration, $O$ granulation tissue or varying grades of fibrous $N$ stenosis. The limitations of bronchoscopy, how- స్ట ever, in this connection should be clearly realized. 응 It is only possible to examine the main and lobar 0 branches and the orifices of the first segmental divisions. Many lesions will thus remain beyond $\stackrel{?}{+}$ the range of bronchoscopic vision. Broncho- $\square$ graphy, particularly with the new water soluble iodine preparations, often gives invaluable informa- 


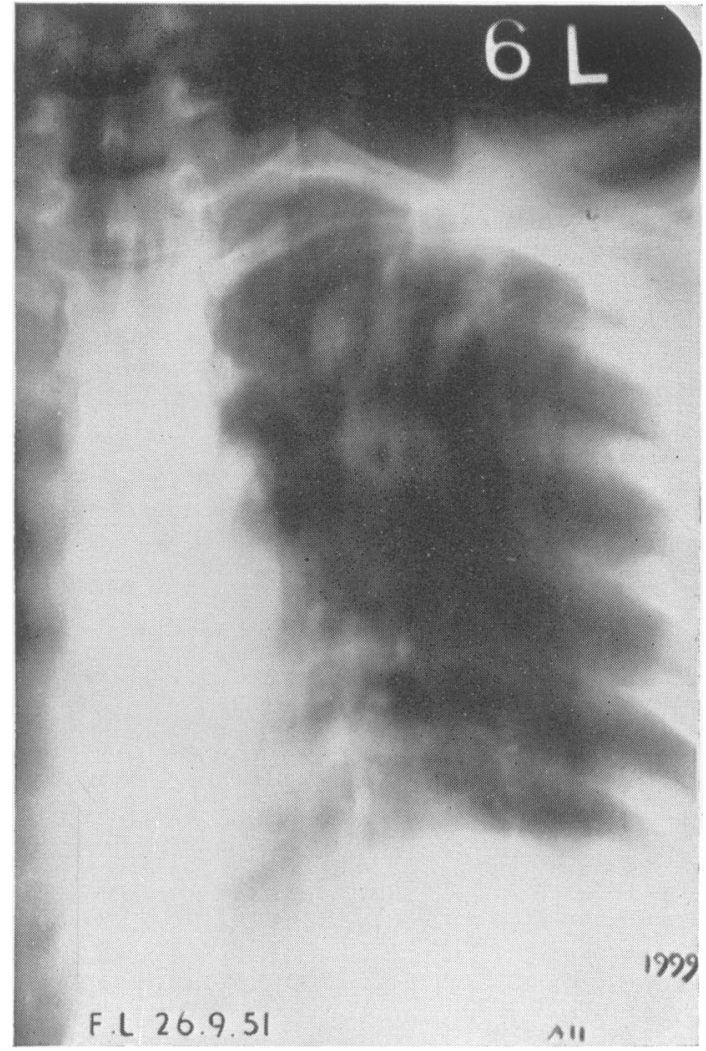

FIG. 2 (a)

FIG. 2.- 'Cold abscess' of bronchus, or 'bronchial pyocoele' treated by segmental resection. (a) P.A. tomographs showing 2 solid rounded foci one of which shows 2 small translucen cies. (b) Lateral film reveals that the lesion lies in the posterior segment of the upper lobe. (c) Specimen showing 2 bronchial abscesses. In both instances the bronchus immediately proximal to the lesion was stenosed. These lesions represent dilated bronchi which are filled with caseous material.

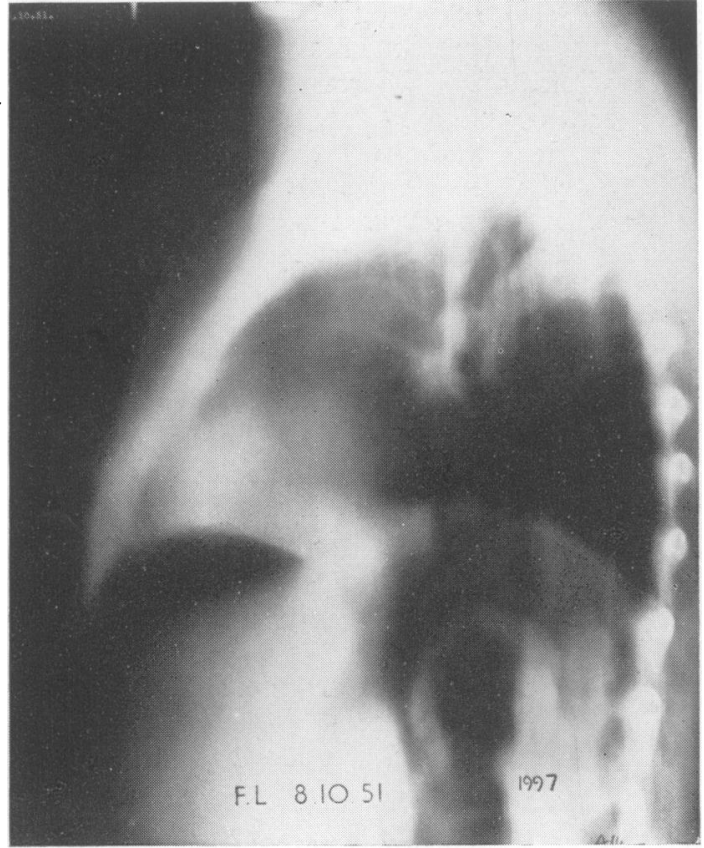

FIG. 2 (b)

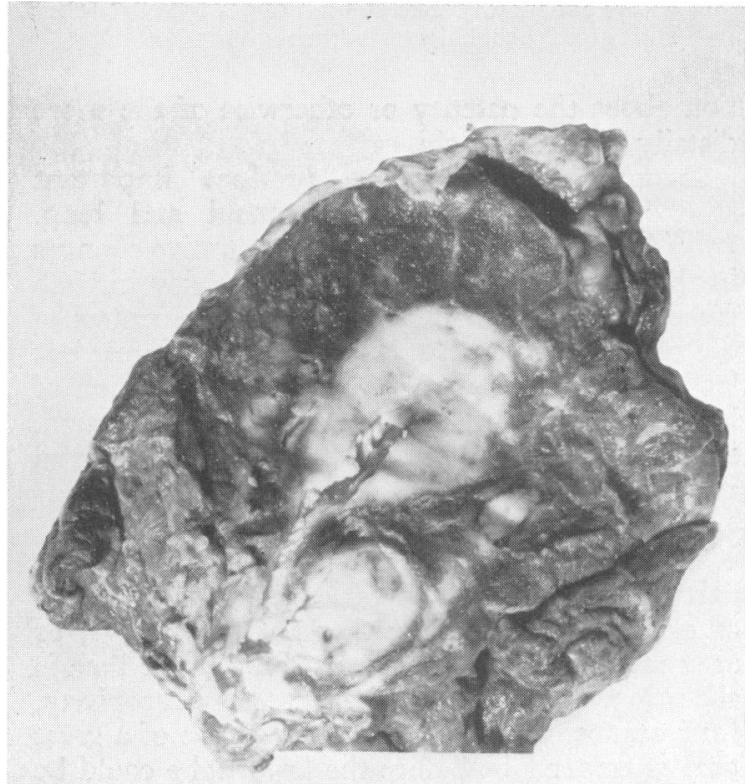

FIG. 2 (c) 


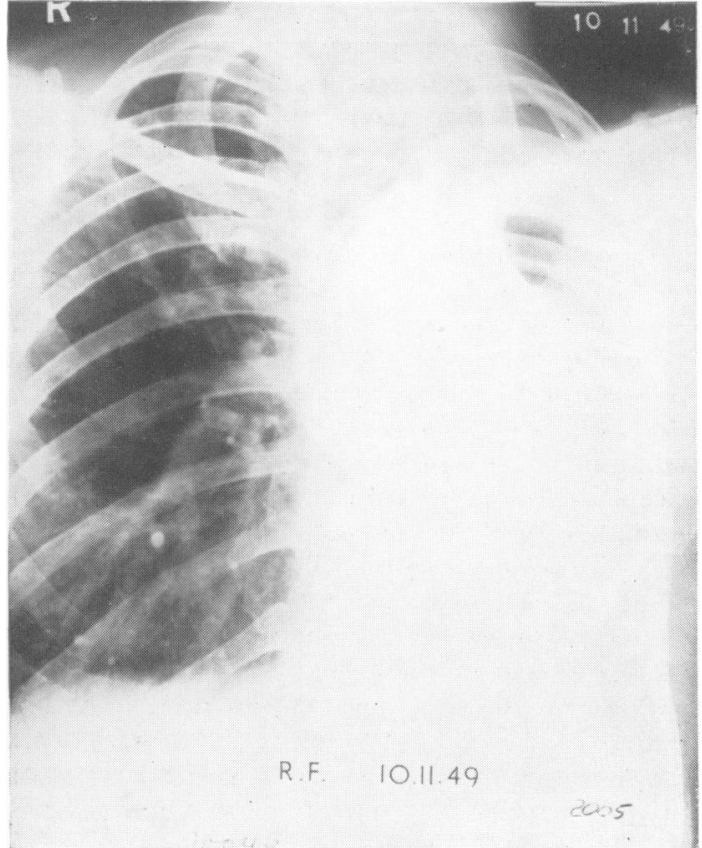

FIG. 3 (a)

FIG. 3.-Ten year history of progressive tuberculosis. Ieft pneumonectomy (1948) for destroyed lung. Poor risk case with much purulent sputum. Alive and well 5 years after operation. (a) P.A. film before operation shows a completely opaque left lung containing a large apical cavity. (b) Specimen shows a markedly stenosed left upper lobe bronchus and gross bronchiectasis throughout the lung.

tion about the patency or otherwise of the more distally placed bronchi.

Broncho-stenotic lesions produce important secondary changes in the bronchi and lung. Bronchiectasis is common and suppurative changes in the lung are frequent. Thoracoplasty in such cases may result in partial or complete control of the tuberculous element in the involved area, but leaves behind a destroyed and useless portion of lung which may well lead to symptoms of cough, sputum and haemoptysis, severe enough to demand a secondary resection.

\section{Lower Lobe Disease}

Tuberculous cavities in the lower lobe are particularly difficult to treat as the usual methods of collapse therapy are either not as effective, or are more destructive of normal lung tissue than is the case with upper lobe disease. A thoracoplasty, for instance, would involve the collapse of a great deal of upper lobe before the lower lobe could be relaxed. Phrenic paralysis combined with a pneumoperitoneum gave hopes of success when first introduced but experience has shown that this combination will close only about 50 per cent.

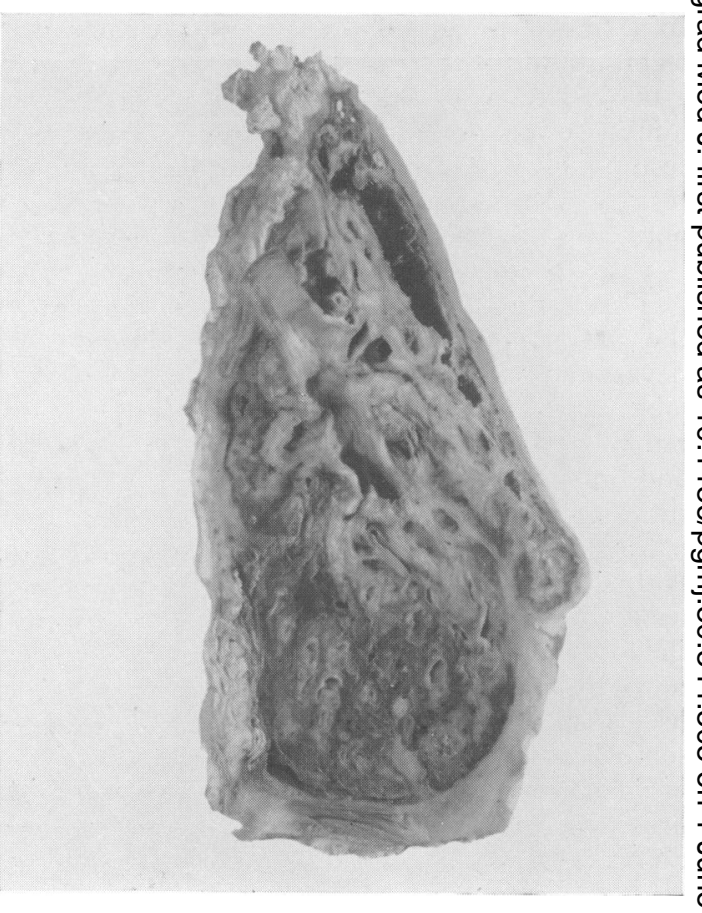

FIG. 3 (b)

of cavities, and even of those closed a proportio eventually re-open.

For lower lobe cavities, the majority are agreed that resection is the method of choice provided the disease is not involving other parts of the lung. $\cong$ As regards the surgical problems, lower lobectomy $\vec{B}$ is more favourable than upper lobectomy as it is 3 easier to obliterate the residual pleural space by elevation of the diaphragm. Lower lobe disease, however, is of ten associated with significant disease $\overline{0}$ elsewhere in the lung which may well render resection undesirable, and in these circumstances, other methods such as thoracoplasty, localizedplombage, cavity drainage or a combination of 0 국 pneumothorax, pneumoperitoneum and phrenic paralysis may be advocated.

4. Failed Collapse Therapy

Failure of an artificial pneumothorax, thoracoplasty or plombage to close a tuberculous cavity $N_{0}$ after a reasonable interval of time demands further $N$ action. If efficient collapse therapy has not been 0 successful after about 9 months, and if during this $\omega$ time, chemotherapy and postural retention has $\widetilde{\sigma}$ been employed to encourage closure, it is safe to assume that the method is unlikely to succeed with $\mathbb{D}$ the further passage of time. If one form of collapse therapy has failed it is unlikely that other ${ }^{\circ}$ methods will succeed, and under these circum- $\overrightarrow{\mathbb{D}}$ stances it is usual to recommend resection. After $\frac{?}{?}$ 
an artificial pneumothorax there are no special technical difficulties but the removal of a lobe after a thoracoplasty or a plomb is technically a more difficult operation but one which gives satisfactory results and presents no problems concerning the residual dead space which is already well nigh obliterated by the previous collapse procedure.

5. Destroyed Lobe or Lung. (Figure 3.)

This is the term applied to the condition where the tuberculous disease has swept through and devastated a complete anatomical unit such as a lobe or lung. Little or no functioning lung remains whilst the bronchi are dilated and inert and often the seat of secondary infection. Somctimes the tuberculous element has burnt itself out leaving a bronchiectatic and fibrotic lung. Although collapse therapy in such cases has been used with success in the past and is occasionally the only practicable form of treatment today it inevitably leaves behind a bronchiectatic and useless lung which may later give rise to troublesome symptoms.

Such cases are better treated by resection provided the remaining lung tissue is sound. So often, however, the rest of the lung is involved so that resection is too hazardous, and in these cases collapse therapy is not only justified but a reasonable alternative.

\section{Anteriorly Placed Cavities}

Cavities in the anterior segment of the upper lobe, in the lingula or in the middle lobe are difficult to treat by thoracoplasty owing to their position. Such cavities are undoubtedly best treated by resection whenever it is practicable.

7. Cold Abscess of Bronchus. (Figure 2.)

Recently attention has been drawn to a lesion produced initially by a tuberculous focus in the wall of a small bronchus leading to obstruction of the latter either from a caseous mass or from secondary cicatricial contraction. As a result the bronchus distal to the obstruction becomes filled with caseous material and has been termed a ' cold abscess of the bronchus,' or 'bronchial pyocoele.' These lesions can often be recognised clinically and radiologically. Their clinical course is one with little systemic disturbance and toxicity but is punctuated with exacerbations associated with a positive sputum. Radiologically they present as peripherally situated oval shadows with a thickened stem bronchus leading towards the hilar region. One or more small translucent areas are often seen in the opacity on tomography.

It seems unlikely that such lesions could be adequately and certainly controlled by collapse therapy and resection is advised in those cases which are not quiescent.

\section{Tuberculous Bronchiectasis}

Three separate conditions are included in this group. (I) Bronchiectasis which has incidentally become secondarily involved by tuberculosis presumably by lighting up a latent tuberculous focus in its midst. (2) True tuberculous bronchiectasis due to extensive involvement of the bronchi by tuberculous disease. (3) Isolated or patchy bronchial dilatations which frequently occur in the vicinity of tuberculous lesions. The first two can only be adequately treated by resection if symptoms are present although conservative measures such as postural drainage and the antibiotics may help to reduce symptoms. The latter is an almost accidential bronchographic finding and is very unlikely to give rise to symptoms (except occasional minimal bleeding) and will not demand special treatment.

\section{Tuberculous Empyema}

The whole approach to the treatment of tuberculous empyema has changed since the introduction of streptomycin and the practice of resections. Previously reliance was placed upon repeated aspirations or total thoracoplasty to obliterate the empyema space whilst external drainage was required for those with secondary infection or a bronchopleural fistula.

Now it is universally agreed that major surgery gives far quicker and better results and has completely replaced the former régime except in certain special instances.

A tuberculous empyema which is not associated with uncontrolled or active disease in the underlying lung should be excised (pleurectomy, decortication) but if there is disease in the lung underneath this also should be excised with the empyema (pleuro-lobectomy, pleuro-pneumonectomy). Thoracoplasty is only employed in those cases with significant disease on the opposite side.

Although the operation is a severe one which is sometimes accompanied by serious blood loss, the results give relief of infection and toxicity and the restoration of lung function are well worth the added risks.

\section{Relative Indications for Resection}

In this group the indications for resection are more debatable and not so universally accepted as those mentioned in the previous section. Much of the uncertainty is due to lack of information and the position should be clarified in time.

\section{Segmental Lesions}

The earlier manifestations of pulmonary tuberculosis are often limited to one or two segments with the remainder of the lung free from disease. Such cases are usually treated initially with rest and chemotherapy followed by some form of surgical treatment. Such a policy has been evolved from the following observations and experiences:- (a) Although rest and chemo- 
therapy may produce dramatic improvement residual nodules frequently remain. (b) Where such nodules persist, relapse is common on returning to full activities. (c) Examination of excised 'quiescent' nodules often reveals the presence of viable and virulent tubercle bacilli.

There is thus an increasing tendency to recommend prophylactic procedures to prevent such relapses. Surgical collapse therapy in the form of thoracoplasty, plombage or extrapleurals are entirely satistactory but probably result in a greater loss of lung function than would be the case with a segmental resection. There is an increasing tendency to carry out resections in such cases.

\section{Cavities in the Apex of the Lower Lobe}

I have already indicated that lesions in the lower lobe are best treated by resection as collapse therapy is difficult to apply. Cavities in the apex of the lower lobe are, however, a little more favourably situated for collapse measures and thoracoplasties or localized plombage operations are often practicable. The former, however, results in the collapse and consequent loss of function in the greater part of the upper lobe. This is an advantage if the latter is diseased but if not it is an unnecessary sacrifice of functioning lung tissue. Localized plombage conserves more lung tissue but is a method which has not yet been given an adequate trial although results are encouraging. Resection has much to offer, particularly for isolated lesions in the apex of the lobe. Where, however, these are combined with upper lobe disease which would necessitate an upper lobectomy in addition, it is doubtful if there is much to be gained from the functional point of view as in such cases it will be necessary to do a thoracoplasty in addition to the resection in order to close the residual space.

\section{Acute Tuberculosis. (Figure 4.)}

The majority of cases of acute pneumonic or broncho-pneumonic tuberculosis respond adequately and often rapidly to rest and chemotherapy. Occasionally the response is disappointingly slow or incomplete, and in such cases resection should be considered as it gives good results provided the disease is limited to a lobe or a lung. Some consider that resection is too radical and risky a procedure and prefer to continue for long periods with medical treatment. They argue that resolution may eventually occur and it is not possible to forecast how much functioning lung tissue might be salvaged by these means. The individual case must be judged on its merits but the possibilities of surgery for localized disease with toxic manifestations not responding to chemotherapy must be borne in mind.

\section{Tension Cavities}

Cavities are formed by a combination of ulcera- tion and inflation and the majority of establishedo cavities are thus strictly 'tension' cavities and? pressures of the gases within them are positive The term 'tension' or " distension' cavities ${ }_{c}{ }^{2}$ however, refers to large thin walled oval or spherical cavities usually found in the upper lobes or apex of lower lobe. Such cavities are more difficult to close with a thoracoplasty than otherso although closure can often be obtained if thes operation is carefully and logically carried out $\stackrel{\mathbb{Q}}{\complement}$ This may often mean, however, an extensiven collapse with considerable encroachment on normal lung tissue and an almost total thoraco-? plasty may often be required. In such cases $\overrightarrow{\vec{\omega}}$ resection has the advantage of leaving the remain-o ing lung free to expand and thus ensuring bette? subsequent function.

5. Fibro-caseous upper lobe disease with oiw without cavitation provides the real bone of contention in the argument of resection versus $\$$ collapse therapy. This particular type andw distribution of disease is probably more commono than most other varieties and in the past has giverf good results with thoracoplasty. Complicationsare few and the percentage of unclosed cavities of significient (i.e. symptom producing) bron? chiectasis is small.

The protagonists of resection maintain that excision of these areas is advisable to remove infected foci, that resection even when combirede with a small thoracoplasty is less deforming ands causes less reduction of lung function than $\overline{\mathrm{D}}$ standard thoracoplasty and there is no risk of unclosed cavities or bronchiectasis. Against this, however, resection does carry a small risk of th $\vec{E}$ production of a broncho-pleural fistula or apica $\bar{B}$ empyema, both complications of some severity and importance.

No clear cut plan is available in such cases and only time and the long-term progress of the resection group will perhaps help to clarif the issue.

\section{Pre-Requisites for Resection}

It will have become obvious from the earlief discussion on indications for resection that in many cases there is little to choose between. surgical collapse therapy and resection and that ing those cases where a resection is indicated it is not always practicable because of lesions elsewhere in the lung.

The following factors will influence the decisior in such cases.

(a) Disease in the Remaining Lung Tissue

After resection the residual lung tissue on the same or opposite side not only carries on the whole respiratory function but by its over-expansiors partly compensates for the loss of lung substance्巳 


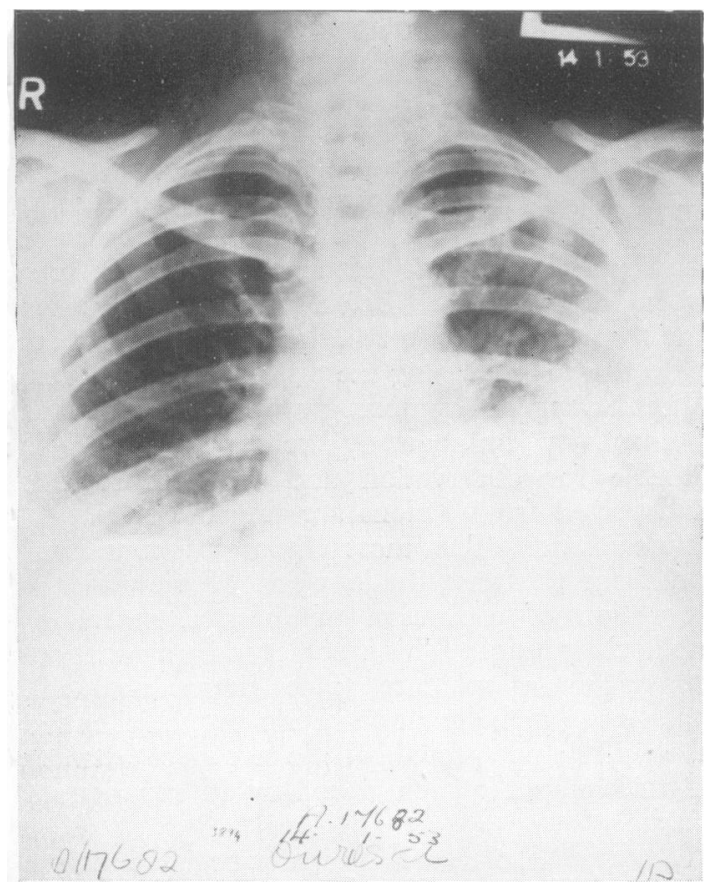

FIG. 4 (a)

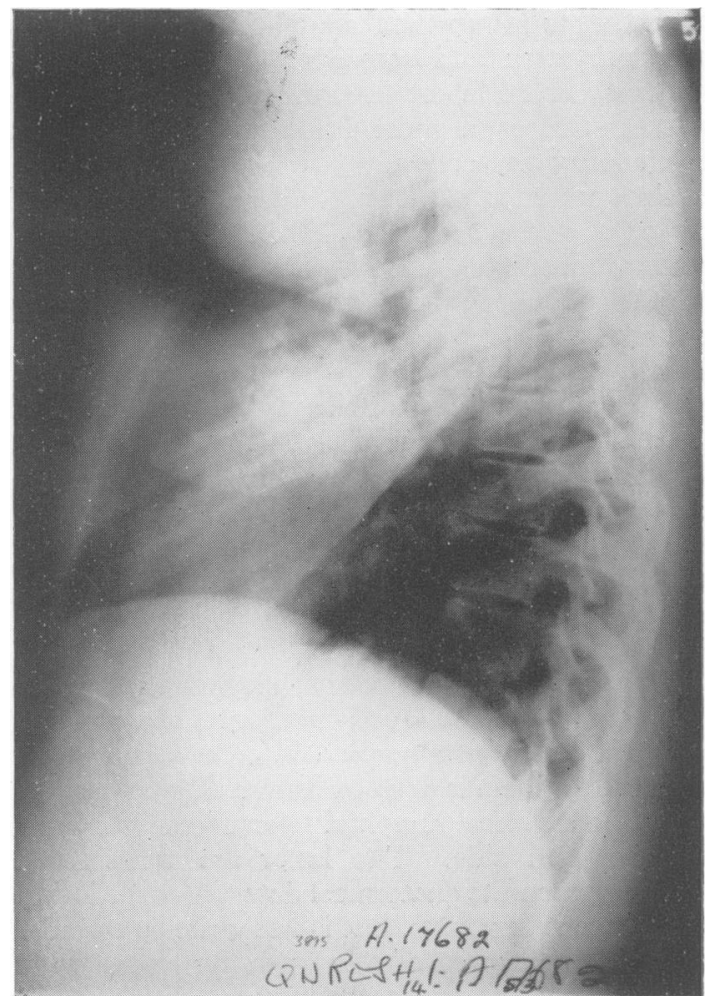

FIG. ${ }^{-} 4(\mathrm{~b})$

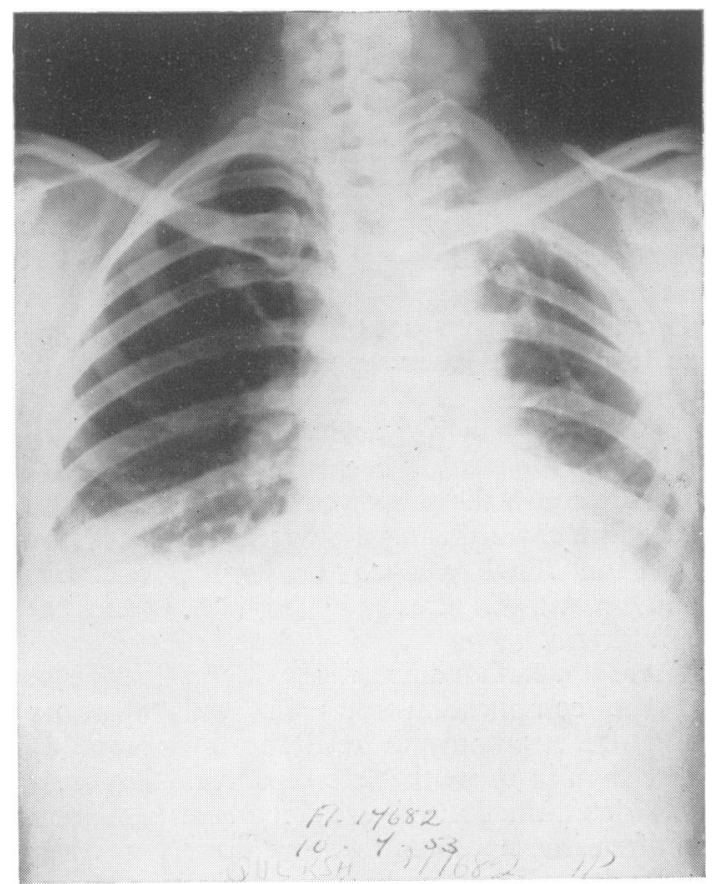

Fig. 4 (c)

Fig. 4.-Acute pneumonic disease with cavitation. Left upper lobectomy, April, 1953, 3 months after acute spread. Space reduction by apical thoracoplasty. (a) P.A. film after acute exacerbation showing a cavity at the left apex and extensive infiltration at the base. (b) Left lateral film showing disease limited to upper lobe. (c) P.A. film 3 months after upper lobectomy. The lower lobe occupies approximately its normal position. 
and helps to fill up the residual dead space. This over-expansion and associated increased ventilation may cause latent tuberculous foci to become active; this is a most important consideration in planning a resection.

The presence of active disease, particularly if cavitated, in the lung to be left behind is a definite contra-indication to resection at that time. Should it subsequently be controlled either by chemotherapy, the passage of time, or by surgery then a resection could be re-considered. As a very rough guide to stability, one year without radiological change is regarded as a reliable index.

Should nodulation be present in the lung or lobe which remains-even though it is regarded as quiescent, it is wise to prevent that lobe or lung from over-expanding by methods which will be outlined later.

It will thus be apparent that a very careful assessment of the whole of both lungs is necessary before operation. Good radiographs are an essential, and tomography almost equally so. Most information is derived from A.P. tomographs of the contralateral lung and lateral tomographs of the operation side. The latter are valuable in obtaining accurate anatomical data about the lesion and adjacent segments, but is not quite so suitable for a routine survey of a lung owing to the wide variations in soft tissue shadows.

\section{(b) Activity of Disease}

One of the most difficult tasks confronting the phthisiologist is the assessment of activity of disease. Fever, a raised E.S.R., loss of weight and radiological changes in serial films give certain limited help but the decision often remains remarkably difficult. If active disease is suspected it is advisable to delay operation and continue with rest and chemotherapy. This applies even more forcibly if active disease is seen in the bronchi at bronchoscopy.

If active disease is not obvious it is only necessary to give a short preoperative course of streptomycin.

\section{(c) Streptomycin Sensitivity}

Mention has already been made of the value of streptomycin in the preparation for operation and its use as a cover during the operation. It is rare for an individual to reach the stage of operative treatment without already having had a course of streptomycin.

There is a certain amount of evidence to suggest that the complication rates in patients whose bacilli are streptomycin resistant is appreciably higher than in those that are sensitive. Unfortunately determination of resistance takes time and it is often impracticable to delay surgery pending the results. With intelligent anticipation, however, such reports may often be obtained in time and may well have an important bearing on the decision as to the type of surgery to be carried out?

\section{The Operation}

Preparation. Careful preparation for operation. should never be neglected as the operation is usually one of election and not an emergencyo Short periods of rest and chemotherapy (I to 30 weeks) are advisable beforehand during which time sepis elsewhere (teeth, nose and bronchi) can $\mathbb{D}$ be dealt with, breathing exercises can be institutech and coughing instructions given. Such a period? of inactivity immediately before operation is. valuable for observation and will ensure that unsuspected fresh lesions are unmasked.

Anaesthesia. The most important considera-O tion from the anaesthetic point of view is the prevention of aspiration of infected secretionsi into healthy lung. This is achieved by a variety of ways such as posture (face down position), endo-bronchial balloons and endo-bronchial cuffedw tubes. There is probably little to choose betweenor the various methods employed by an experiencedo operator.

Operative Technique. It is not possible to give details of technique here but certain important苘 principles should be mentioned.

(a) Avoidance of contamination of the pleugalces cavity.

(b) Extrapleural enucleation of adherent are

(c) Avoidance of a long bronchial stump.

(d) Careful bronchial closure.

(e) In any segmental or lobar resection avoid-气 ance of transgressing tuberculous disease. $\stackrel{\Phi}{\Omega}$ Never leave a raw surface which might be $\vec{O}$ doubtfully involved in tuberculous 3 disease.

As regards the extent of the resection the important considerations are to remove the offending lesions with an adequate safe margin and 3 . in an approved anatomical manner and yet? conserve as much normal lung tissue as possible. At the same time unnecessary risks should not beo taken in leaving behind doubtfully active areas of disease. This latter consideration is one which음 may often necessitate the removal of more than was $>$ intended and should always be borne in mind을 before operation. This is one of the inherent disadvantages of resection therapy.

The most conservative form of removal is the $N$ excision of an isolated nodule (nodulectomy) $\mathbb{\omega}$ followed by wedge resection for very limited peripherally situated foci, segmental resectiono lobectomy and pneumonectomy. In any one case various combinations of the above may be ${ }^{\text {? }}$ necessary to eradicate the obvious lesions.

After removal of the involved tissue the surgeon $\frac{\overrightarrow{+}}{\Phi}$ is faced with the problems of rapid elimination 


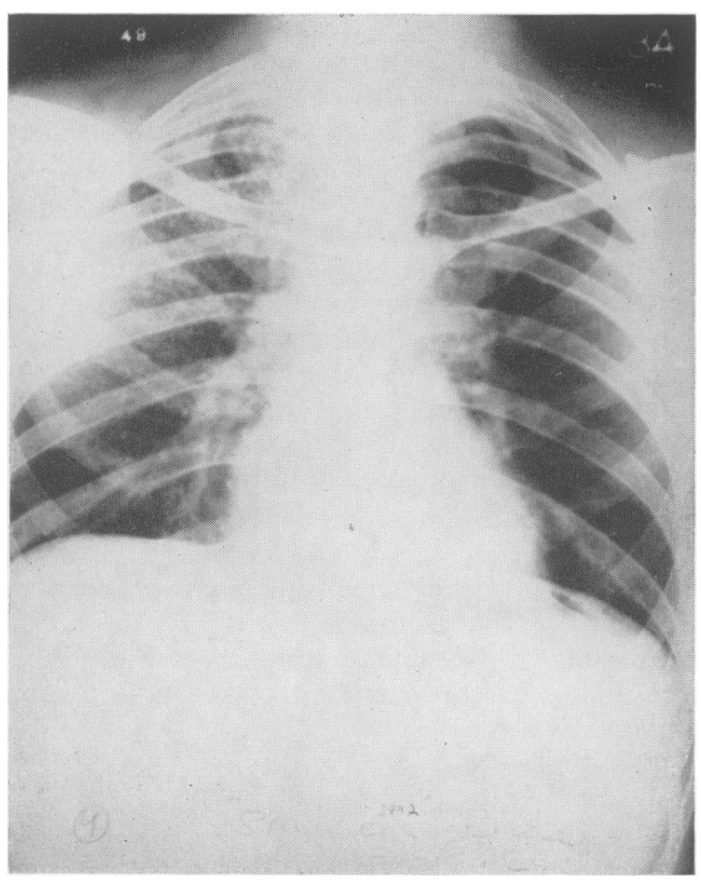

Fig. 5 (a)

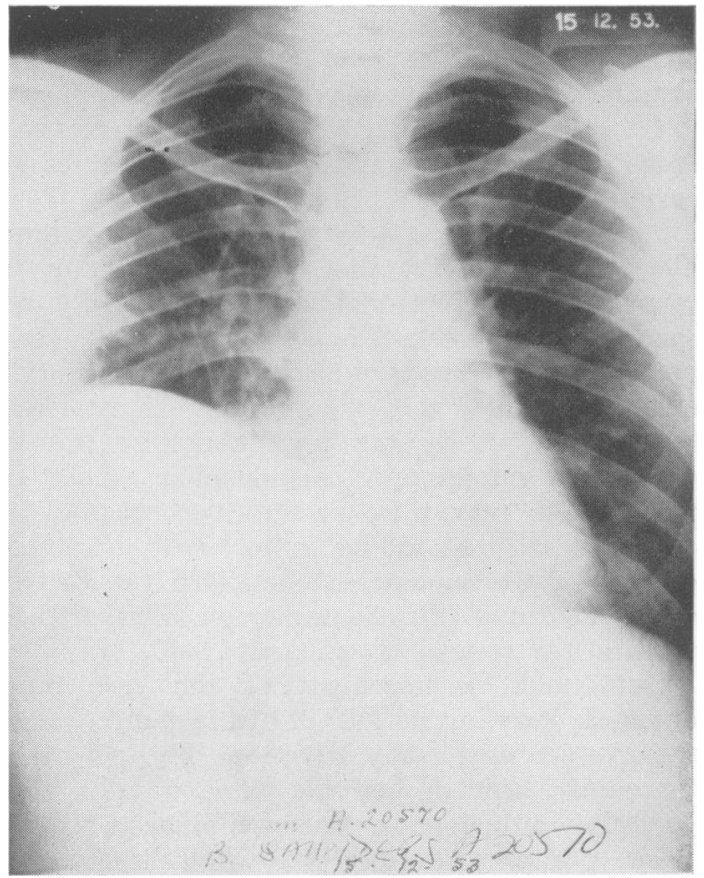

Fig. 5 (b)

Fig. 5.-Chronic cavitated disease in right upper lobe. Right upper lobectomy, May, 1953. Space reduction with phrenic paralysis and temporary pneumoperitoneum. (a) P.A. film before operation showing infiltration and cavitation in the right upper lobe. (b) P.A. film 6 months after operation. The lower and middle lobes now occupy the upper half of the chest.

of the residual dead space without undue overexpansion of the remaining lung tissue.

In this connection the following observations are relevant:-

I. Continuous and efficient removal of all air and fluid exuding from raw lung surfaces must be effected by carefully planned tube drainage with or without the use of suction.

2. Small losses of tissue can usually be compensated for by expansion of adjacent lung segments and no further steps will be necessary.

3. Loss of a lobe or smaller loses associated with residual infiltration must be compensated for by a space reducing measure, such as phrenic paralysis, a pneumoperitoneum either singly or in combination (Figure 5) or an apical thoracoplasty (Figure 4) or plombage. In the case of an upper lobe satisfactory space reduction is obtained by removing portions of the 2nd and 3 rd ribs and stripping the pleura from the under surface of the Ist rib but not removing it. Both thoracoplasty and plombage can be performed at the conclusion of the resection without placing undue strain on the individual. Phrenic paralysis can readily be done at the time of the resection but a pneumoperitoneum is best induced a few days before operation to allow the patient to become accommodated to the presence of air in the abdomen. 4. After pneumonectomy, space reduction is often adequately achieved by elevating the paralysed diaphragm with a pneumoperitoneum and relying on mediastinal migration and chest wall contraction to do the rest. If these factors do not appear to be adequate after 2 to 3 weeks, or if it appears that the opposite lung is expanding unduly, then a controlling thoracoplasty is advisable. Thoracoplasty done at the same time as pneumonectomy is advocated by some and can be safely carried out in patients whose condition is good but in others it is a greatly added burden and is probably more safely deferred for a few weeks.

\section{Post-Operative Management and Complications}

In the post-operative care of patients after resection there are two overwhelmingly important considerations.

I. Early and complete obliteration of pleural dead space. Mention has already been made about steps to achieve this end taken during the operation. After operation efficient tube function must be maintained at all times with water seal or 
suction according to taste. In this way air and fluid is removed as soon as it collects in the pleural cavity and the remaining lung is encouraged to fill the residual space. Failure to obliterate this space is one of the most important causes of empyemata.

2. Removal of tracheo-bronchial secretions. There is no doubt that the accumulation of secretions in the bronchial tree is usually responsible for atelectasis and for many of the early post-operative spreads of disease. After operation efficient coughing is very important. A vital role here is played by the physiotherapist but all concerned, nurses and doctors alike, must be prepared to play their part in encouraging the cough. Frequent rolling in bed with breathing exercises at the same time, inhalations, expectorant cough mixtures and chemotherapy all have their place in the control of secretions.

Subsequent management of the case must obviously vary but periods of bed rest and chemotherapy are universally advised. The patient is frequently kept in bed for three months after operation although he may be allowed up for toilet purposes during the latter six weeks. Chemotherapy is continued for at least six weeks after operation, sometimes for much longer periods. A further period of 3 to 4 months slow convalescence is required and this is best carried out in a sanatorium before returning home and to work.

The three most impotant complications apart from those which one may encounter after any form of surgery are :-

I. Atelectasis

Mention has already been made about the prevention of this complication. Sometimes the development of atelectasis is quite silent and the diagnosis only made radiologically. More often, however, there is a febrile disturbance associated with difficult expectoration and reduction of sputum. The latter is usually thick and sticky. Physical signs consist of mediastinal displacement towards the affected side, dullness and absent air entry or bronchial breathing. The X-ray shows a homogenous opacity in the place of the aerated lung.

Treatment must be vigorous and initially directed at the encouragement of coughing and expectoration. With modern chemotherapy further action is not desperately urgent provided symptoms are minimal, but if the patient is toxic, dyspnoeic or distressed and aeration is not achieved early then bronchoscopic aspiration should be carried out without delay. In very sick individuals this can be carried out in the ward with a minimum of disturbance.
2. Broncho-Pleural Fistula.
(See Table
2.)

This is undoubtedly the most dreaded of the complications of resection and carries a high $\frac{2}{3}$ mortality and morbidity. Persistent leakage of air is frequent immediately after lobectomies and $\mathrm{d}_{\llcorner}^{\varrho}$ segmental resections from the raw lung surface but sealing usually occurs within a few days. Thes chief danger of such a leak is that it will prevento full expansion of the lung to fill the dead space.으 True broncho-pleural fistulae usually occure between the first and third week after operation $\stackrel{\square}{\varnothing}$ and are almost always due to infection in theo stump. The complication can be reduced by careful bronchial suturing, the avoidance of a long. stump and the avoidance of tight sutures.

Histological examination of the bronchus at the site of resection shows tuberculous foci in theo majority, and it is surprising that tuberculous 3 . infection of the stump is not more frequently. encountered. No doubt streptomycin used be- $\omega$ fore and after the operation plays an important preventative role.

The development of a fistula is often heralded by fever, and the production of blood-stained sputum ${ }^{\supset}$ similar to the fluid present in the pleural cavity. $\overrightarrow{\vec{c}}$ If the leakage is small, control is often possible by repeated daily aspirations combined with active ${ }^{\overparen{D}}$ chemotherapy and healing may occur. Usuabye however, drainage will be required and the resultant empyema cavity and the fistula may tafed long to heal and may well require further surgicis measures to close it.

Early thoracotomy and re-suture of the bronchuso is advocated by some, but the results of such measures are not very encouraging.

3. Empyema. (See Table 2.)

There are two factors which together are? responsible for the majority of empyemata. One. is failure to obliterate the pleural dead spaceo rapidly and completely, and the second is the development of a broncho-pleural fistula.

The importance of maintaining efficient tube drainage with the removal of all air and fluid as ite appears has already been mentioned. It is usually unwise to leave a tube in the pleural cavitys for longer than 4 to 5 days owing to the risk of introducing infection along the tube track? Following the removal of the tube any further collection of air or fluid should be aspirated or if excessive a new tube should be introduced at different site.

It is probably superfluous to mention that every care should be taken to avoid contamination of the pleural cavity at the time of operation, though it is surprising how well minor degrees of soiling are tolerated.

Established pleural infection calls for regula aspirations of the pleural cavity with the removaf 
of as much fluid as possible and the use of intrapleural antibiotics. Aspirations should be repeated daily until the infection subsides and the fluid no longer accumulates. Should aspirations fail to control the infection, pleural drainage should be carried out.

An established empyema will often take a very long time to heal and chronicity is common; this is particularly so in the case of an apical empyema which may follow upper lobectomy and in such cases an early thoracoplasty is often indicated. Late Complications

The future status of resection in the treatment of pulmonary tuberculosis very largely depends on the question of late complications and particularly on the long-term stability of the patients.

Reactivation of old disease or the appearances of fresh infiltration does occur from time to time and is more likely to appear where lung tissue has been unduly expanded especially when small foci were present in this area of lung. (Hence the advisability of preventing over-distension by various measures already out-lined.) Such lesions often respond well to rest and antibiotics though occasionally surgical measures are required. (See Table 2.)

Tuberculous ulceration of the bronchial stump occurs from time to time and may be responsible for haemoptysis and a positive sputum. Healing can often be achieved by further administration of antibiotics.

Late tuberculous infection of a pneumonectomy space and late empyemata occur very occasionally and are notoriously difficult to treat at this stage. Results of Operation

As it is only about five years since resections for tuberculosis were carried out in any number it is not possible to give a really long-term follow-up report at this stage. However, it is possible to out-line the immediate complications and results and give a medium-term follow-up on a moderate series.

The results of any series of surgical operations are meaningless unless one knows much of the hackground against which the cases are treated. Not only must one know the various indications for operation but something of the approach of both the surgeon and his physicians to surgery. Carefully selected cases should have a negligible mortality and morbidity. But if only such cases are treated then many deserving patients will be denied the prospect of cure or amelioration of their symptoms even though the risks are high. A surgeon's figures will therefore depend very largely at what point he draws the line of inoperability. The figures given here represent all my own personal cases and are designed mainly to indicate trends in the treatment and demonstrate that even with a fairly radical out-look the mortality rates and morbidity figures are not excessive or prohibitive.

In Table $\mathrm{I}$ the cases have been grouped according to an assessment of the operative risks into good, fair and poor risks based on a consideration of the extent and type of the disease, age, coexisting conditions such as emphysema, bronchitis, amyloid disease, diabetes, etc. The figures indicate a preponderance of poor risk cases in the pneumonectomy group and none amongst the segmental resections. It also demonstrated the much higher mortality in this group but here a salvage rate of 50 per cent. is well worth while as many of these cases were quite desperate risks with a very limited prospect without surgery.

Table 2 shows the hospital and late mortality for the three types of resection and the more serious complication rates. Of the ten late deaths after pneumonectomy four were not directly attributable to pulmonary tuberculosis but this high figure indicates the greater risk that a patient with one lung carries especially with regard to non-specific pulmonary infections.

Table 3 illustrates a decreasing number of pneumonectomies and corresponding increases in lobectomies and segmental resections from I948 to 1952. This is largely due to a more conservative surgical approach and the conservation of normal lung tissue whenever possible.

Improvement in these figures should be expected with increasing knowledge and experience particularly as regards the selection of cases.

\section{Conclusion}

It will thus be seen that the removal of tuberculous lung tissue can be safely carried out without undue mortality or morbidity. The operation has opened up fresh fields and has added a very powerful weapon to those already combating this disease. The final status of this type of surgery has yet to be agreed; that it will be an important one can hardly be denied. 